NOUVELLE

\section{Hippocampe, striatum et séquences}

Geneviève Albouy, Pierre Maquet
G. Albouy: Centre de Recherches du Cyclotron, Université de Liège, B30, Sart Tilman, B-4000 Liège, Belgique. Université de Lyon; Lyon, France. CNRS UMR 5167 ; Lyon, France. Inserm U821; Lyon, France. albouy@sommeil.univ-lyonl.fr P. Maquet : Centre de Recherches du Cyclotron ; Université de Liège ; Liège, Belgique. Departement de Neurologie, CHU Sart Tilman; Liège, Belgique.

motrices restent encore mal connus. II semblerait que les phases précoces de l'apprentissage soient prises en charge par des réseaux cérébello-corticaux tandis que la rétention à long terme des mémoires de séquences motrices utiliserait des réseaux striato-corticaux [7].

\section{Hippocampe, striatum et séquences}

Le but de notre étude [8] consistait à élucider les corrélats cérébraux de la consolidation d'un apprentissage implicite de séquences oculomotrices. Nous avons donc enregistré de jeunes sujets volontaires sains en imagerie par résonance magnétique fonctionnelle (IRMf) lors d'un apprentissage de séquences oculomotrices [9]. Les participants ont été retestés 30 minutes, 5 heures ou 24 heures (incluant une période de sommeil nocturne) après l'entraînement initial (Figure 1A). Les corrélats cérébraux de la consolidation de la trace mnésique ont été caractérisés de

La mémoire procédurale :

apprentissage de séquences

La mémoire procédurale constitue l'un des principaux systèmes de mémoire chez l'homme. Ce type d'apprentissage mène, par exemple, à l'acquisition graduelle d'une habileté nouvelle à exécuter un mouvement complexe (apprentissage moteur). L'apprentissage de séquences motrices constitue l'un des paradigmes d'apprentissage procédural les plus étudiés. Les séquences motrices font partie intégrante d'un grand nombre d'activités de la vie quotidienne comme écrire, parler, tricoter, conduire une voiture ou jouer d'un instrument de musique. Lors de l'apprentissage de séquences motrices, il est couramment observé que les performances s'améliorent significativement au cours de la première session d'entrâ̂nement, au fur et à mesure de la pratique.

A Protocole expérimental

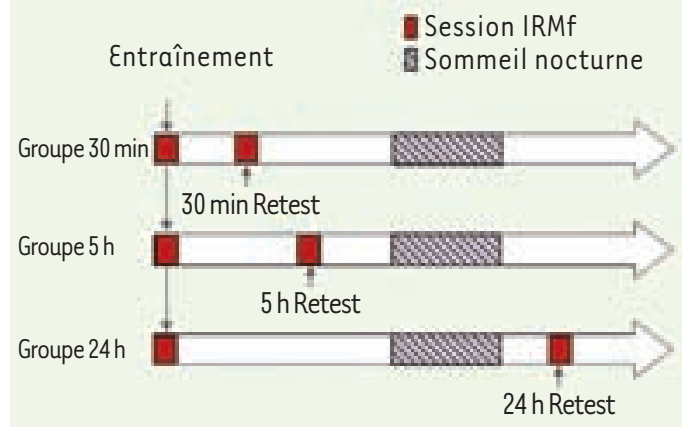

B Tâche d'apprentissage

(3)

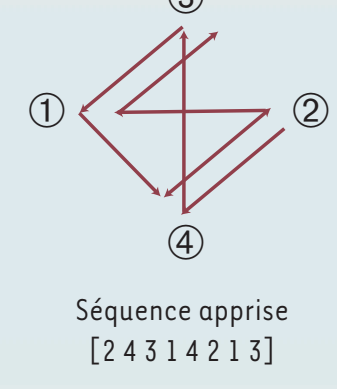

Figure 1. Protocole expérimental et tâche d'apprentissage. A. Tous les volontaires ont été entraînés à la tâche dans le scanner. Ils ont alors été repartis en 3 groupes expérimentaux, selon la date du retest ultérieur dans le scanner (30 minutes, 5 heures ou 24 heures après la fin de l'entraînement). B. La trajectoire du point dans la tâche d'apprentissage suit toujours la même séquence de déplacement qui est apprise de manière implicite par les participants. 


\section{A Réponses cérébrales pendant l'entraînement}

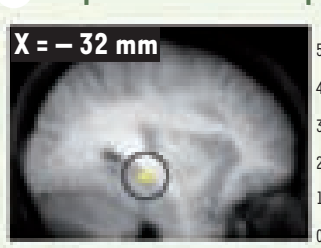

Hippocampe

B Réponses cérébrales au retest

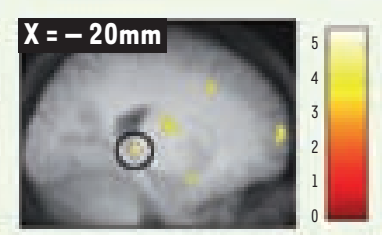

Hippocampe

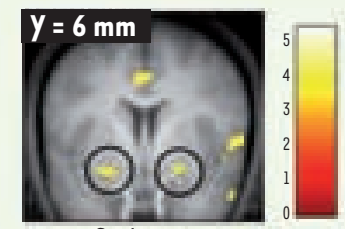

Striatum

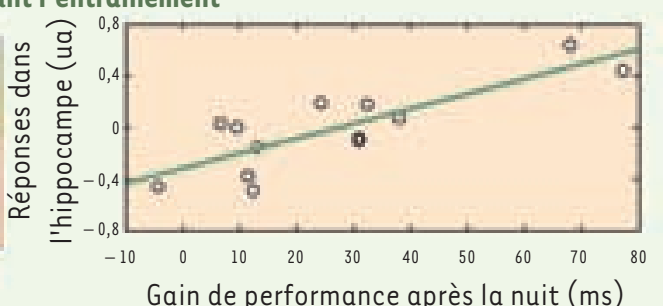

Gain de performance après la nuit (ms)
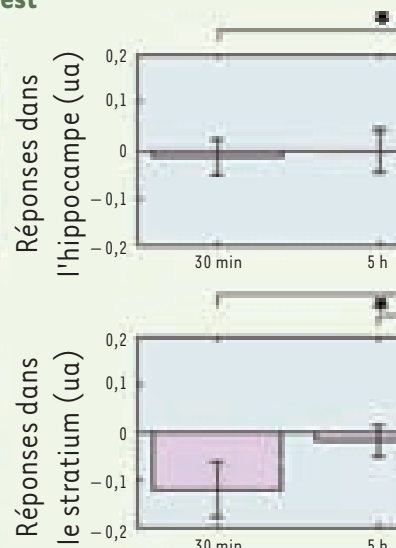

ㄸ- -0

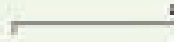

$\longrightarrow$
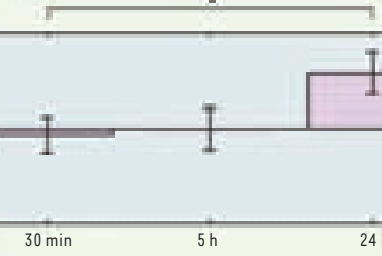

$30 \mathrm{~min}$
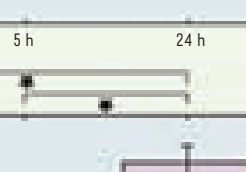

Figure 2. Résultats de l'imagerie par résonance magnétique fonctionnelle. A. Les réponses cérébrales dans l'hippocampe pendant l'entraînement sont linéairement reliées aux gains de performance observés après la nuit de sommeil. $\boldsymbol{B}$. Les réponses cérébrales dans l'hippocampe et le striatum sont plus importantes lors du retest à 24 heures que lors de celui effectué à 30 minutes ou à 5 heures après l'entraînement. ua : unités arbitraires.

manière indirecte par la modification des réponses cérébrales observées lors du retest. Pendant la tâche d'apprentissage, les participants avaient l'instruction de suivre des yeux un point qui se déplaçait à l'écran dans le but de détecter un bref changement de sa couleur. Cependant, à l'insu des sujets, le mouvement du point suivait toujours la même séquence de déplacement (séquence apprise) tout au long du test (Figure 1B). Nos résultats montrent que la performance des sujets (temps de réaction oculaire pour atteindre le point) s'améliore au cours de la pratique de la tâche indiquant que les volontaires ont appris inconsciemment la séquence de déplacement du point [9]. Une amélioration des performances est observée au cours de la journée (retests à 30 minutes et à 5 heures) mais, de manière intéressante, cette amélioration devient spécifique de la séquence apprise seulement après une nuit de sommeil (retest à 24 heures).

Au niveau cérébral, comme attendu, les réponses caractérisant l'apprentissage

sont observées à la fois dans l'hippocampe et le striatum. Cependant, la principale découverte de cette expérience réside en l'observation que ces réponses sont proportionnelles aux gains de performance observés après la nuit (24 heures après l'apprentissage, Figure $2 A$ ) mais pas au cours de la journée ( 30 minutes ou 5 heures après l'apprentissage). Le recrutement précoce de l'hippocampe et du striatum lors de l'apprentissage de séquences motrices prédit donc le gain de performance observé après la nuit. L'activation de ces régions lors de l'apprentissage semble donc profondément influencer la consolidation nocturne des mémoires motrices. $\varepsilon$ n effet, c'est seulement après la nuit que les réponses dans l'hippocampe et le striatum sont plus importantes comparées aux retests ayant eu lieu pendant la journée (Figure 2B). Finalement, les interactions fonctionnelles entre ces deux structures primordiales pour l'apprentissage semblent se modifier au cours des phénomènes de consolidation. En effet, l'interaction compétitive observée pendant l'entraînement entre l'hippocampe et le striatum semble devenir coopérative après la nuit, permettant d'optimiser le comportement moteur.

\section{Conclusions}

Nos résultats montrent que l'hippocampe, région du lobe médio-temporal jusqu'ici décrit surtout dans la consolidation de la seule mémoire déclarative, joue un rôle primordial dans la consolidation des mémoires procédurales. Les réponses dans l'hippocampe et le striatum conditionnent les phénomènes de consolidation observés après la nuit qui sont associés à des changements d'interaction fonctionnelle entre ces aires cérébrales permettant d'optimiser les mouvements séquentiels moteurs. $\diamond$ Hippocampus, striatum and sequences

\section{RÉFÉRENCES}

1. Cohen NJ, Squire LR. Preserved learning and retention of pattern-analyzing skill in amnesia : dissociation of knowing how and knowing that. Science $1980 ; 210$ : 207-10.

2. Poldrack RA, Clark J, Pare-Blagoev EJ, et al. Interactive memory systems in the human brain. Nature 2001 ; 414 : 546-50.

3. Schendan HE, Searl MM, Melrose RJ, Stern CE. An FMRI study of the role of the medial temporal lobe in implicit and explicit sequence learning. Neuron 2003 ; 37 : 1013-25.

4. Karni A, Meyer G, Jezzard P, et al. Functional MRI evidence for adult motor cortex plasticity during motor skill learning. Nature 1995 ; 377 : 155-8.

5. Maquet $P$, Laureys $S$, Peigneux $P$, et al. Experiencedependent changes in cerebral activation during human REM sleep. Nat Neurosci 2000 ; 3 : 831-6.

6. Peigneux $P$, Laureys $S$, Fuchs $S$, et al. Learned material content and acquisition level modulate cerebral reactivation during posttraining rapid-eyemovements sleep. Neuroimage $2003 ; 20: 125-34$.

7. Doyon J, Penhune V, Ungerleider LG. Distinct contribution of the cortico-striatal and corticocerebellar systems to motor skill learning. Neuropsychologia $2003 ; 41:$ 252-62.

8. Albouy, G, Sterpenich V, Balteau $\varepsilon$, et al. Both the hippocampus and striatum are involved in consolidation of motor sequence memory. Neuron 2008; 58: 261-72.

9. Albouy G, Ruby P, Phillips C, et al. Implicit oculomotor sequence learning in humans: time course of offline processing. Brain Res 2006 ; 1090 : 163-71.

10. Vigot R. La dépression synaptique à long terme: un mécanisme pour la mémoire et l'apprentissage au niveau du cervelet. Med Sci (Paris) 2003 ; 19: 437-41. 